\section{Reliability of drop size from multi-dose eye drop bottles: is it cause for concern?}

EMMA J. GERMAN, MARK A. HURST, DIANA WOOD

\begin{abstract}
Purpose Responses to topically applied ocular drugs vary between patients. The volume of drug instilled is of particular importance as one of many sources of response variation, but the reliability of drop volume from eye drop bottles is unknown. Hence, the repeatability of drop volume and factors affecting this for a variety of drug manufacturers were considered in this study.
\end{abstract}

Methods Nineteen bottles, one from each primary manufacturer in the UK, were examined. The mass of all drops expelled from each bottle was measured with respect to the bottle type, handling angle, drop number, drug and concentration. The accuracy (repeatability and trueness) of drops from each bottle was also evaluated.

Results Drop volume varied significantly between drug manufacturers, ranging from 33.8 $\mu \mathrm{l}$ to $63.4 \mu \mathrm{l}$. The handling angle of the bottle also influenced drop volume, with angles less than $60^{\circ}$ giving smaller drops. Drop number exhibited no significant effect upon drop volume. However, the drug type and its concentration did significantly affect the volume of the drop expelled from the bottle, with higher concentrations giving rise to larger drops. Repeatability coefficients across the range of bottles varied between $\pm 2.24 \mu l$ and $\pm 10.76 \mu l$ (mean $\pm 5.07 \mu \mathrm{l})$.

Conclusions It is well reported that drug volume instilled has a significant effect on the degree of response. However, there are currently no official regulations concerning eye drop volume in either the UK or the USA. Since drop volume has been shown to vary significantly depending upon a variety of factors, it may be appropriate that the regulatory bodies consider the consequences of variable drop size.

Key words Drop volume, Multi-dose eye drop bottles, Ophthalmic drug delivery
Currently, ocular drugs are most frequently applied in the form of drops, with many of the commonly used pharmacopoeias (for example Martindale, the British Pharmacopoeia and the Merck Index) recommending practitioners to instil 1 or 2 drops as required. However, as highlighted by the Pharmaceutical Codex, ${ }^{1}$ there can be 'a considerable degree of imprecision' in the dose administered. This may be a cause for concern since the pharmacological effect of a compound can be greatly influenced by the volume instilled. ${ }^{2,3}$ In general, smaller volumes tend to induce less lacrimation and thus increase the contact time of the drug with the cornea, resulting in an increased response. ${ }^{4}$ An increase in the volume instilled does not necessarily cause an increase in the bioavailability of the drug., ${ }^{5,6}$ Reduced volumes can be obtained by using reduced eye dropper tip dimensions ${ }^{7}$ or by attaching a cannula to the bottle. ${ }^{8}$ Smaller volumes then offer a means by which the likelihood of secondary systemic effects, particularly in young children where the body is immature and low in mass, can be reduced.

Several physical factors may potentially be responsible for variation in drop size, such as the dimensions of the aperture ${ }^{7}$ (Fig. 1), the characteristics of the drug solution (surface tension, ${ }^{7,9}$ viscosity, ${ }^{7}$ density) and handling angle of the container. ${ }^{10}$ Assessment of the repeatability of drop size and of any factors likely to cause increased variation is therefore of great clinical importance. It will highlight any areas where the drop size can be controlled to a higher degree of accuracy and enable better assessment of the influence of the above physical factors on the resultant effect of any topically applied ocular drugs.

Single-use containers, such as Minims, are widely used in clinical ophthalmic practice in the UK. They have the advantages of being sterile, preservative-free, less likely to cause cross-infection and available in a comprehensive range of drugs. Despite this,
E.J. German

M.A. Hurst

Department of Optometry University of Bradford Bradford, UK

D. Wood

Department of Pharmacology University of Bradford Bradford, UK

E.J. German Department of Optometry University of Bradford Richmond Road Bradford

West Yorkshire BD7 1DP UK

Tel: $+44(0) 1274234640$ Fax: +44 (0)1274 235570 e-mail:

e.j.german@bradford.ac.uk

The study was funded by a grant to E.J.G. from the College of Optometrists

Extracts of this work were presented at the Second International Symposium on Experimental and Clinical Ocular Pharmacology and Pharmaceutics, Munich, 1997

Received: 22 June 1998 Accepted in revised form: 23 October 1998 


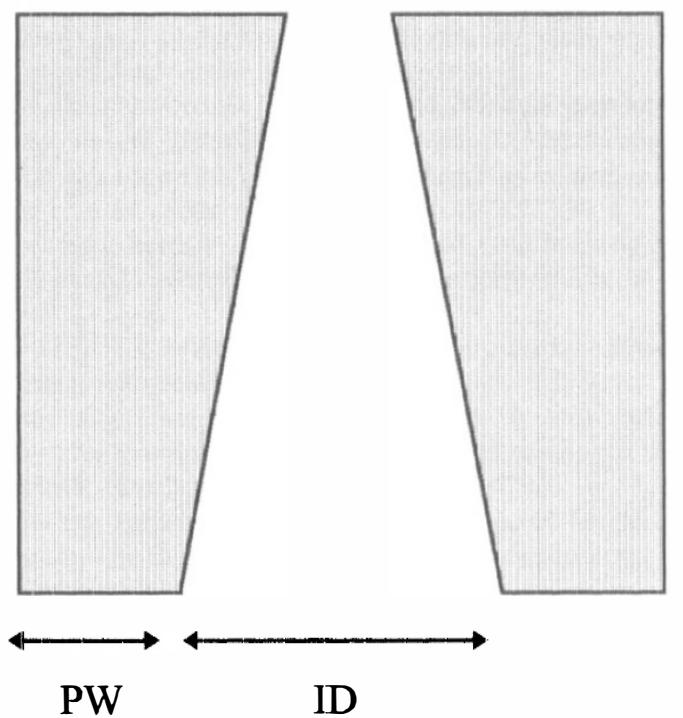

OD

Fig. 1. Basic cross-sectional structure of an eyedropper tip. The inner chamber is separated from the outer chamber by an inner aperture. The outer diameter (OD), platform width (PW) and inner diameter (ID) are critical dimensions in determining the eyedrop's size.

they are not generally used by patients for long-term treatment at home. The most often used alternative is a multi-dose bottle, so called because the bottle is designed to be used either on several patients or given to a single patient to use as part of a therapeutic regimen. Multidose bottles contain preservatives to prolong the shelf life of the active ingredient once opened; these may either cause an adverse reaction or alter the drug action. ${ }^{11}$ In comparison with containers used on single patients, they may also pose a greater risk of cross-contamination. Nevertheless, they are considered to be a more economical choice than single-use containers.
Although strict quality control checks are made on the production of the drugs and their containers, few comprehensive studies have been reported that have assessed the constancy of the size of drop from eye drop containers and any factors that may influence the size of drop. ${ }^{10}$ The main aim of the following study was, therefore, to examine the variation in drop size from a variety of multi-dose bottles and to analyse the influence of several factors on the size of the drops. Furthermore, the accuracy (repeatability and trueness ${ }^{12}$ ) of drop volume was compared between different suppliers of eye drops in the UK.

\section{Materials and methods}

A single pre-filled sample bottle was donated towards the study by each of the $18 \mathrm{UK}$ suppliers of eye drops (Table 1). Alcon donated an additional bottle for investigation of the effect of drug concentration on drop size, and MSD donated a total of 5 bottles to allow investigation of both the variation in drops between bottles from the same supplier and the trueness of the drop size compared with the expected value. Although examination of a larger sample of bottles from each supplier would have indicated whether the performance varied significantly within each batch, one of the main thrusts of the current study was to assess any differences between suppliers rather than to consider individual manufacturer reliability. Initially, the influence of drop number on drop size was investigated by holding each bottle vertically $\left(90^{\circ}\right.$ from the horizontal) and measuring the mass of every drop (i.e. drug) from the bottle using a Mettler AE50 electronic mass balance (accuracy to $0.1 \mathrm{mg}$ ). Once the densities of the drugs were calculated, all mass values were converted to volume measurements for further analysis. Data acquired from the two Alcon bottles was also compared to assess the effect of drug concentration on drop volume.

Table 1. Bottles used in the multi-dose eye drop study

\begin{tabular}{|c|c|c|c|}
\hline Bottle no. & Manufacturer & Trade name & $\%$ drug \\
\hline 1 & Alcon & Mydriacyl & $0.5 \%$ tropicamide \\
\hline 2 & Alcon & Mydriacyl & $1 \%$ tropicamide \\
\hline 3 & Allergan & Betagan & $0.5 \%$ levobunolol \\
\hline 4 & Baker-Norton & Pax-Hypromellose & $0.3 \%$ hypromellose \\
\hline 5 & Boehringer Ingelheim & Mydrilate & $1 \%$ cyclopentolate \\
\hline 6 & Chauvin & Snophenicol & $0.5 \%$ chloramphenicol \\
\hline 7 & CIBA & Otrivine-Antistin & Xylometazoline + antazoline \\
\hline 8 & Dominion Pharma (Cusi) & Cloburate & $0.1 \%$ clobetasone butyrate \\
\hline 9 & Evans & Predsol & $0.5 \%$ prednisolone \\
\hline 10 & Martindale & - & $1 \%$ atropine \\
\hline 11 & MSD & Timoptol & $0.25 \%$ timolol \\
\hline 12 & Novex & Vividrin & $2 \%$ sodium cromoglycate \\
\hline 13 & Parke-Davis & Chloromycetin & $0.5 \%$ chloramphenicol \\
\hline 14 & Rhone Poulenc-Rorer & Brolene & $0.1 \%$ propamidine isethionate \\
\hline 15 & Roche & Genticin & $0.3 \%$ gentamicin \\
\hline 16 & Roussel & Soframycin & Framycetin sulphate \\
\hline 17 & Schering-Plough & - & $0.3 \%$ hypromellose \\
\hline 18 & Squibb & Ophthaine & $0.5 \%$ proxymetacaine \\
\hline 19 & Typharm & Golden Eye & $0.1 \%$ propamidine isethionate \\
\hline
\end{tabular}




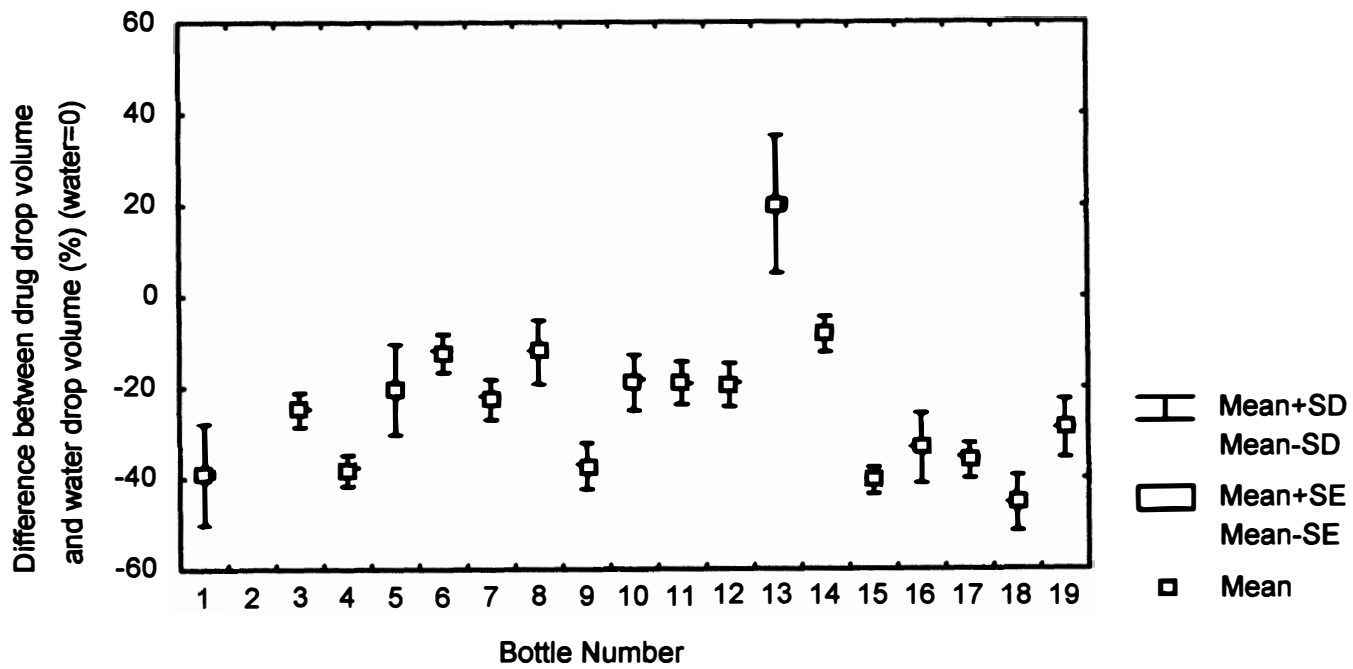

Fig. 2. The change in drop volume occurring when various drugs are expelled from an eyedrop bottle compared with distilled water.

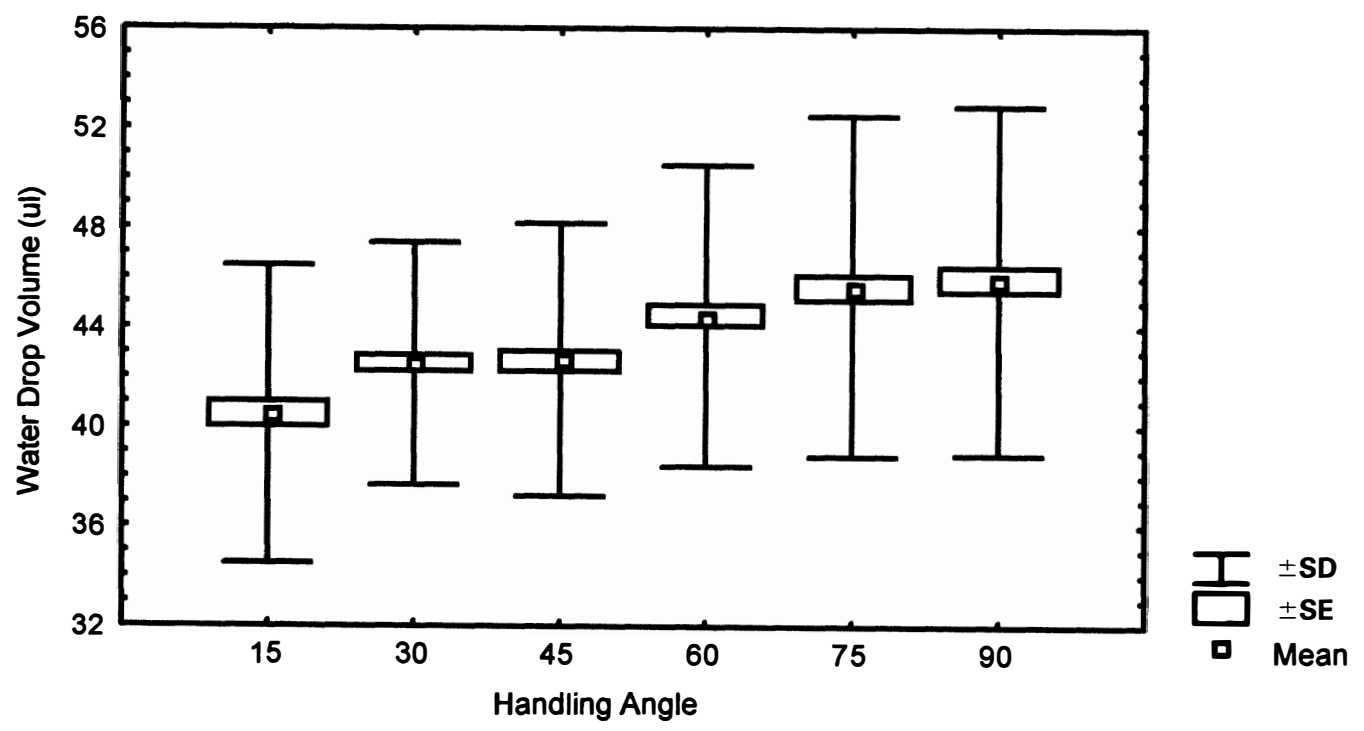

Fig. 3. The effect of altered dropping angle upon water drop size (all 19 bottles).

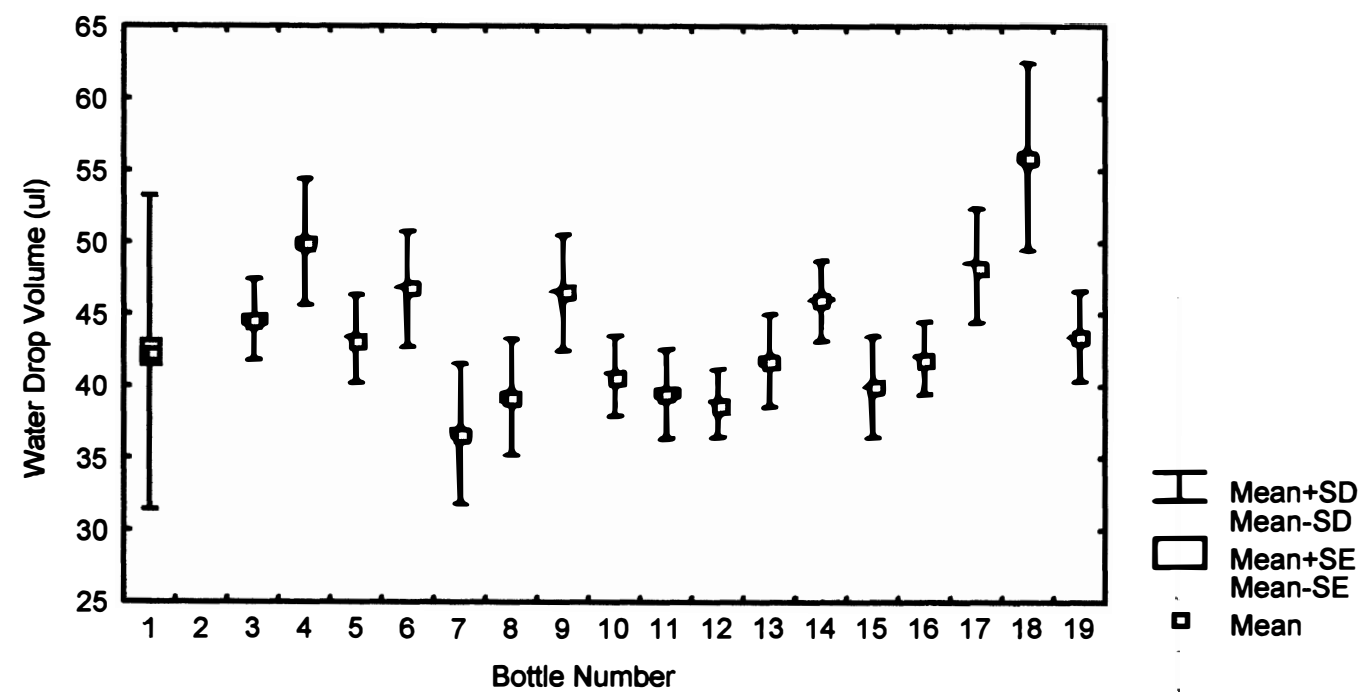

Fig. 4. Variation in water drop volume produced by bottles from different suppliers. 


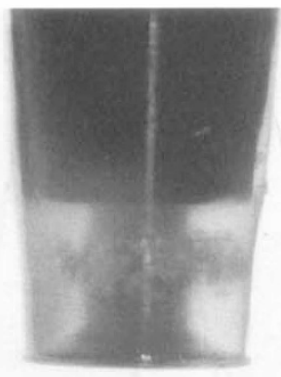

(a)

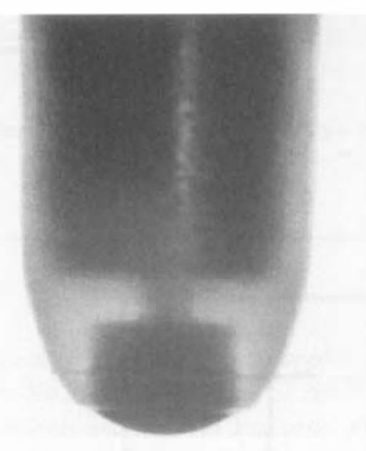

(c)

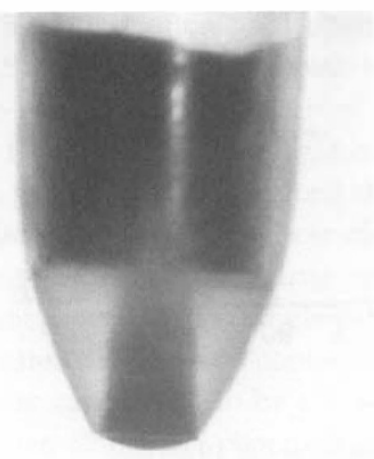

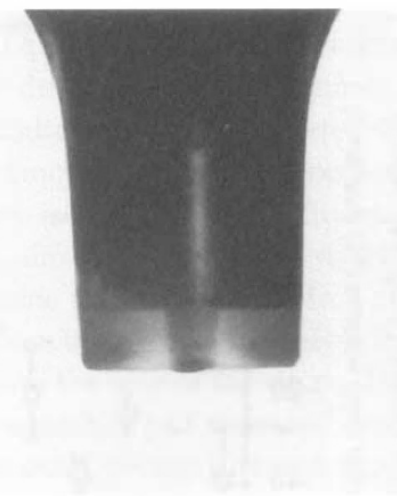

(b)

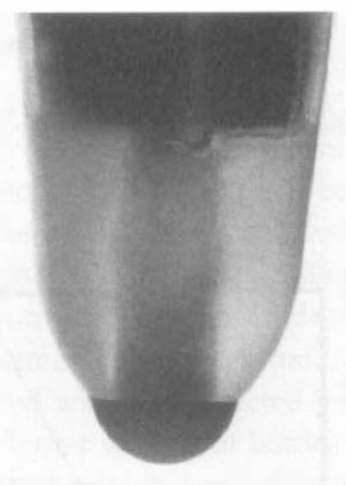

(d)

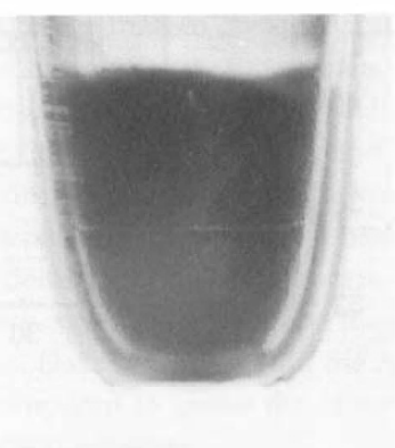

(f)

(e)

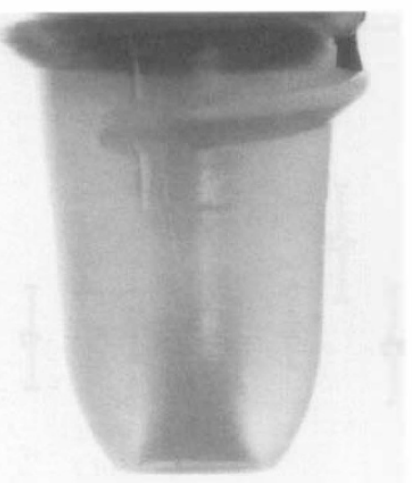

(g)

Fig. 5. Internal nozzle profiles observed amongst the multi-dose bottles studied (nozzles a-g). 


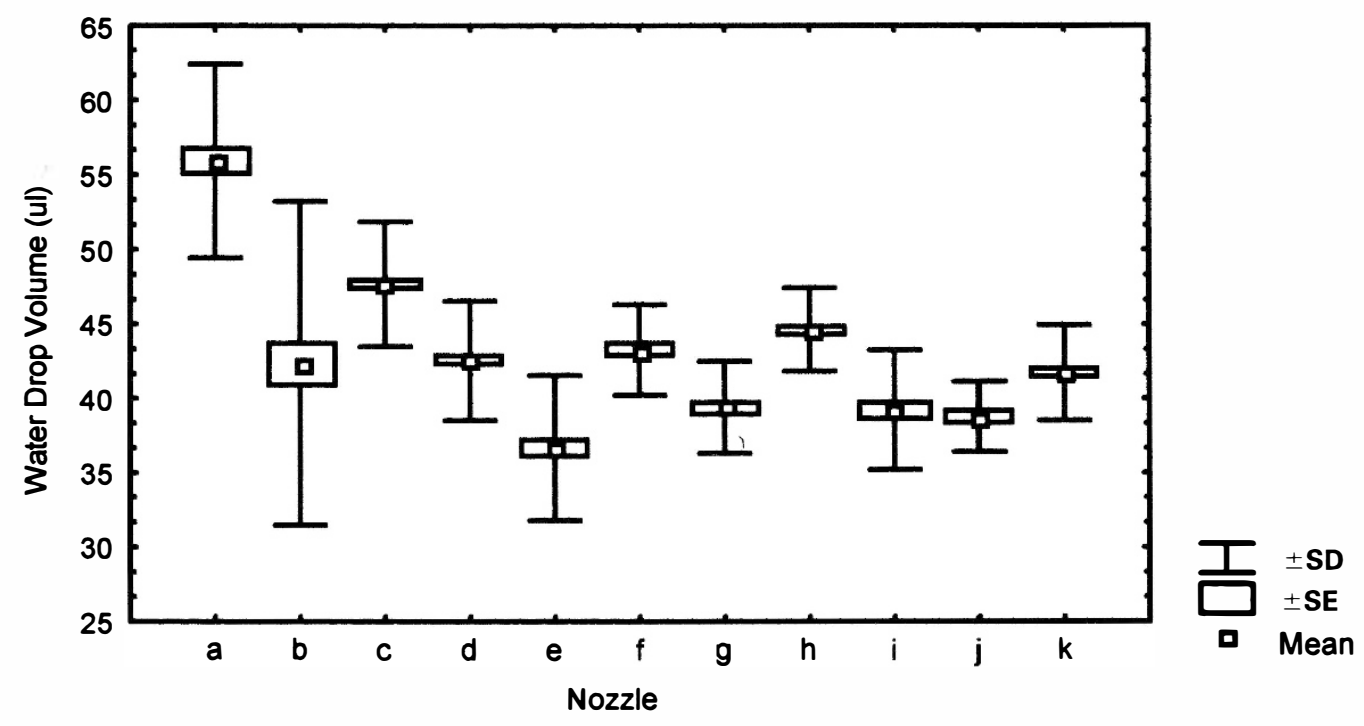

Fig. 6. Influence of the nozzle profile on drop volume.

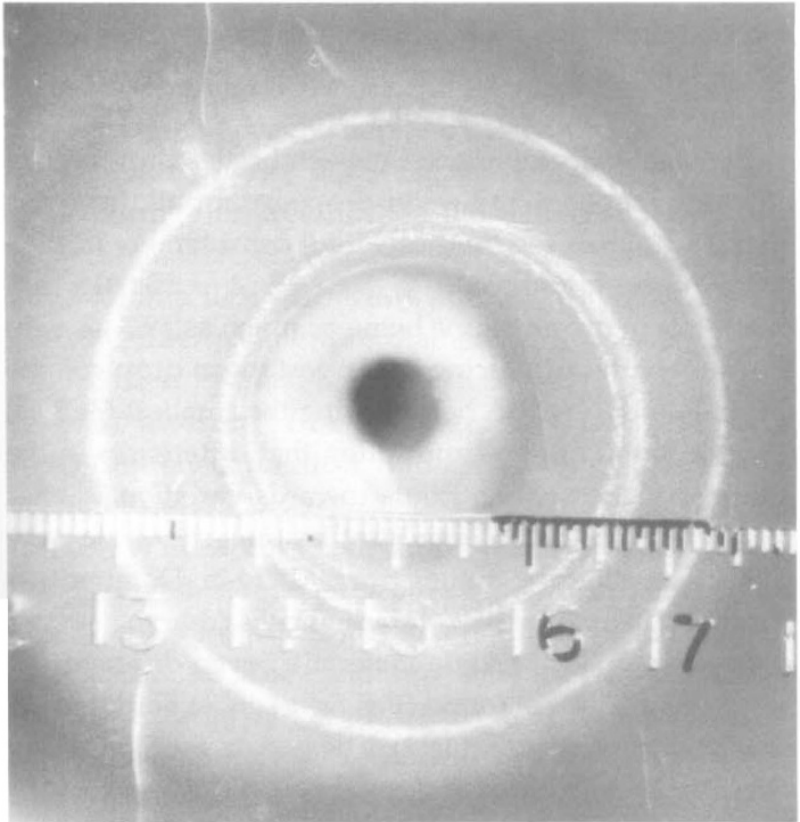

(a)

(b)

Fig. 7. Examples of photographs taken to measure platform width, and inner and outer aperture diameters. (a) Outer aperture and platform width; (b) inner aperture.

To investigate the effect of the dropping/handling angle, each bottle was rinsed and filled with distilled water and manually positioned against an angular scale set by spirit level. Ten drops were expelled at each of six angles $\left(15^{\circ}\right.$, $30^{\circ}, 45^{\circ}, 60^{\circ}, 75^{\circ}$ and $90^{\circ}$ from the horizontal) and their mass measured and converted to volume. To examine the overall effect of different drug types on drop size, data for the drug drops (all held at $90^{\circ}$ ) were compared with water drops from the same bottle also held at $90^{\circ}$. Individual physical parameters, such as surface tension and viscosity, were not measured in this case. To assess any influence of the aperture profile on drop volume, scaled photographs of the limiting apertures were made for each bottle, from which the outer diameter and platform width were directly measured, the inner diameter estimated and then all measurements compared with drop volumes. Photographs of the internal nozzle profile, on the other hand, enabled bottles to be grouped according to the type of manufacturer of the nozzle. The total effect of the nozzle type on drop size was also analysed.

Statistical analysis comprised analysis of variance (ANOVA) with post-hoc comparison of means (Scheffé's test), the Student's $t$-test and Pearson product-moment correlations. The drop number, drug type and concentration, dropping angle, supplier, nozzle type and each aperture dimension were assessed using these tests to determine any significant influence on drop volume. 
Table 2. Nozzle types found on the multi-dose bottles

\begin{tabular}{ll}
\hline Nozzle & Manufacturer \\
\hline$a$ & Squibb \\
$b$ & Alcon \\
$c$ & Baker-Norton, Evans, RPR, Schering-Plough \\
$d$ & Chauvin, Martindale, Roche, Roussel, Typharm \\
$e$ & CIBA \\
$f$ & Boehringer-Ingelheim \\
$g$ & MSD \\
$h^{\text {a }}$ & Allergan \\
$i^{\text {a }}$ & Dominion Pharma (Cusi) \\
$j^{\text {a }}$ & Novex \\
$k^{\text {a }}$ & Parke-Davis \\
\hline
\end{tabular}

${ }^{a}$ Unknown internal profile (opaque nozzle material).

The accuracy of drop volume (defined both by 'repeatability' and 'trueness' ${ }^{12}$ ) was also required. The coefficients of repeatability ${ }^{13}$ were calculated for each supplier. 'Trueness', however, could only be examined for one supplier (MSD) since 'trueness' is a measure of bias between the actual and the expected drop size, and MSD was the only supplier to state the expected drop volume. Four additional sample bottles were acquired from this supplier, and the drug drops measured at a dropping angle of $90^{\circ}$ and compared with the expected drop volume. Where indicated, the results are expressed as the mean \pm standard deviation.

\section{Results}

In the first instance, the data from the drug drops expelled from all 19 bottles suggested that multi-dose bottles produced a drop volume of $32.75 \pm 5.42 \mu \mathrm{l}$, regardless of the different drug solutions used. Since the number of drug drops produced by each bottle exceeded $100($ minimum $=133$, maximum $=439)$, the results were assumed to follow the Normal distribution according to the Central Limit Theorem. ${ }^{14}$ The total number of drops expelled from each bottle had no significant effect on the drop volume in any case (drops 1-300: $F_{299,4292}=0.49$, $p=1.00$; drops $301-439 ; F_{138,402}=0.73, p=0.98$ ).
Where $0.5 \%$ tropicamide was compared with $1 \%$ tropicamide (Alcon 'Mydriacyl'), an increase in drug concentration resulted in a significant increase in drop volume ( $t=-9.88, p=0.001)$. Further examining the concept that drug flow characteristics may differ between completely different drug types, comparison of drug drops with water drops (the first 10 drops expelled at $90^{\circ}$ ) revealed that solutions containing a drug and preservative produced significantly different drop volumes compared with those produced from distilled water $(p<0.005)$. In the majority of cases the drug formed a reduced drop size. Chloramphenicol was the only drug to produce a significantly increased drop size in comparison with water $(t=4.64, p<0.005)$ (Fig. 2).

The influence of dropping angle, assessed by analysis of water drop volumes, highlighted similar results to those obtained from Minims. ${ }^{10}$ Dropping angle did indeed influence drop size $\left(F_{5,107}=21.23, p<0.0001\right)$, although Scheffé's test revealed that there was no significant difference between adjacent angles $(p>0.05)$ and that there was no significant difference between $60^{\circ}$, $75^{\circ}$ and $90^{\circ}(p=0.4)$. However, angles less than $60^{\circ}$ produced significantly smaller drops $(p<0.001)$ (Fig. 3).

To enable a comparison between bottles provided by different suppliers, the water drop size for all dropping angles was analysed. Bottles varied considerably in their drop volumes produced $\left(F_{17,1062}=67.54, p<0.001\right)$, the difference in drop volume being as much as $19.3 \mu \mathrm{l}$, with the Squibb unit producing the largest mean drop volume $(55.9 \mu \mathrm{l})$ and the CIBA bottle giving the smallest $(36.7 \mu \mathrm{l})$ (Fig. 4). Based on the supposition that differences in the nozzle design may contribute towards variation, photographs of the internal profiles of each nozzle were taken, excepting the bottles from Allergan, Dominion Pharma (Cusi), Novex and Parke-Davis (due to completely opaque nozzle material). Seven different nozzle profiles were found (Fig. $5 a-$ g), most bottles using either nozzle $c$ or $d$ (Table 2). The nozzle profile had a significant effect on drop size $\left(F_{10,1069}=92.95, p<0.01\right)$. Nozzle types $a$ and $c$ produced significantly higher drop

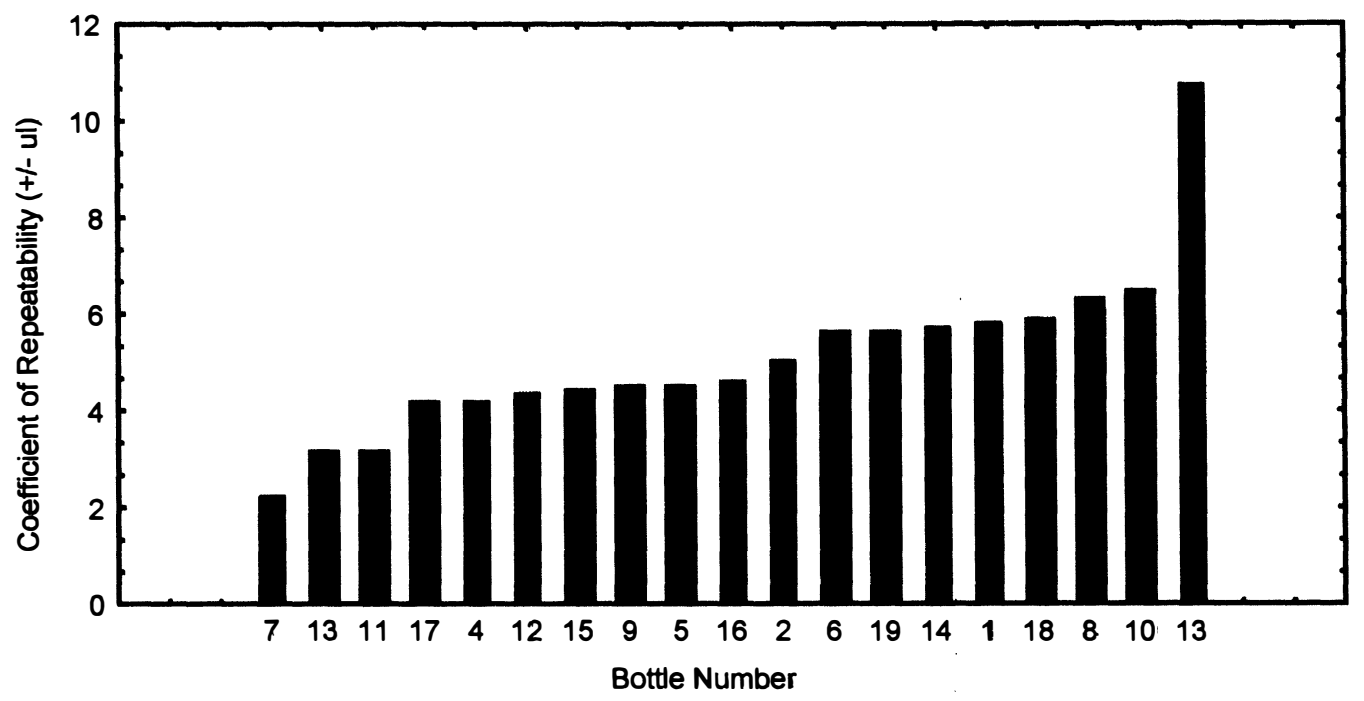

Fig. 8. Range of repeatability of drop volume across different suppliers of eye drops. 


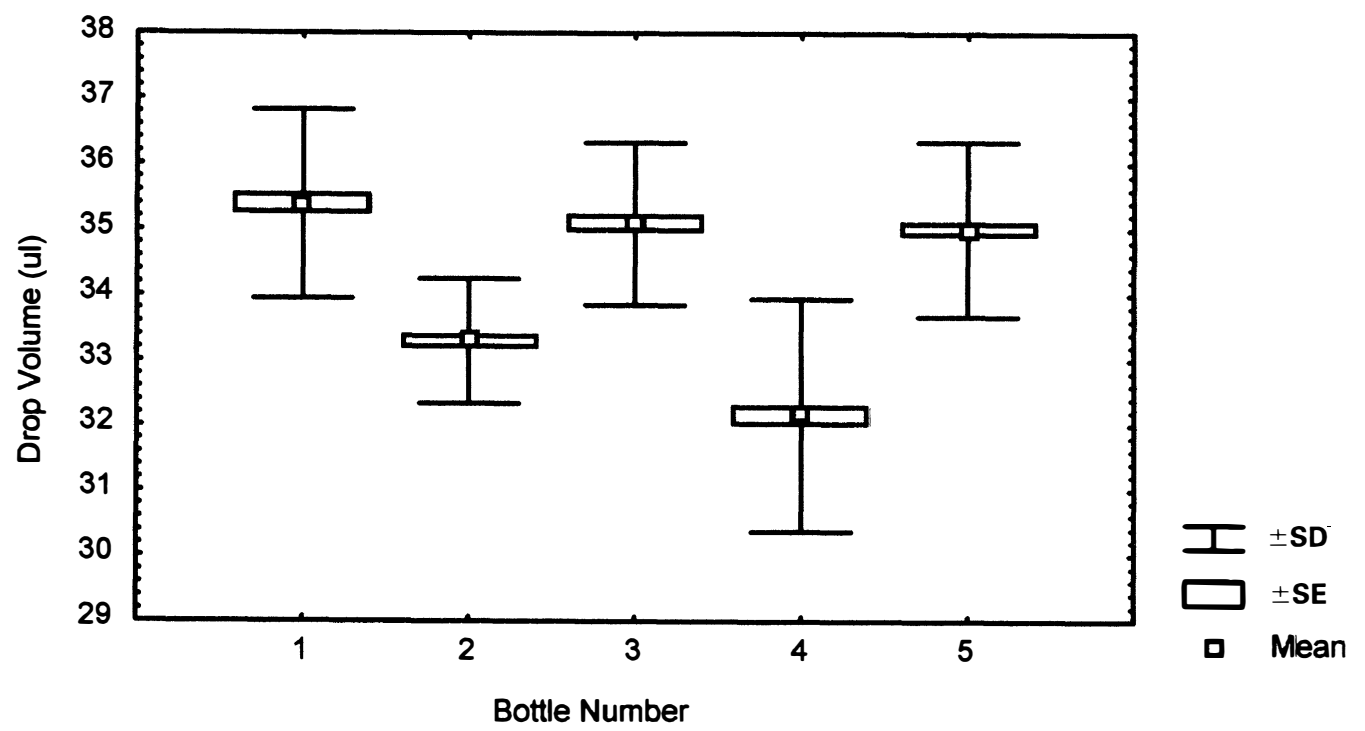

Fig. 9. Variation in the volume of timolol drops produced by different bottles from MSD. The drops from bottles 1,3 and 5 were not significantly different $(p>0.2)$ but bottles 2 and 4 produced significantly smaller drops.

volumes than all others $(p<0.05)$; drop volumes from $b / d / f / h / k$ were approximately the same $(p>0.05)$, as were those from $e / g / i / j(p>0.05)$ (Fig. 6).

Exploring this area further, more precise nozzle dimensions were analysed to assess their influence on drop volume. The platform width $\left(F_{8,831}=63.32\right.$, $p<0.01)$, internal aperture diameter $\left(F_{11,1008}=86.96\right.$, $p<0.01)$ and external aperture diameter $\left(F_{8,1071}=69.59\right.$, $p<0.01$ ), measured from photographs (Fig. 7), all had a significant effect on drop volume, but there was no apparent trend.

Finally, the accuracy of drop volume was compared between bottles obtained from different suppliers. Coefficients of repeatability ${ }^{13}$ varied considerably between bottles. The mean repeatability was $\pm 5.07 \mu \mathrm{l}$, but ranged from $\pm 2.24 \mu \mathrm{l}$ (CIBA) to $\pm 10.76 \mu \mathrm{l}$ (ParkeDavis) (Fig. 8). Trueness, expressed as the bias between actual and expected drop volume, was only calculated for bottles from MSD $(n=5)$. Based upon an expected drop volume of $20 \mu \mathrm{l}$, mean bias was as high as $+14.12 \mu \mathrm{l}$ (or $+70.6 \%$ ). In other words, the drops were $70.6 \%$ larger than intended. In addition, the variation in drop size between the five MSD bottles was statistically significant $\left(F_{4,786}=166.36, p<0.05\right)$, although this may not necessarily be clinically significant (Fig. 9).

\section{Discussion}

This study found that similar parameters as described previously in the Minim study ${ }^{10}$ could also influence drop size significantly in multi-dose bottles. The multidose bottle on average produced a drop of approximately $33 \mu l, 6 \mu l$ larger than a Minim drop. The dropping angle played a significant part in drop size variation, angles less than $60^{\circ}$ giving smaller drops. The drug type may also be influential, presumably because parameters such as the viscosity and surface tension vary among formulations. In contrast to Minims, however, an increase in drug concentration did appear to increase drop volume significantly, although only two bottles were examined. This discrepancy may reflect concentration differences between drugs, phenylephrine (from a Minim) possessing little change in flow characteristics with concentration changes whereas tropicamide is subject to greater modifications with alterations in concentrations. Also conflicting with data acquired from the Minim study ${ }^{10}$ was the effect of drop number on drop volume. No significant changes in drop size were observed as the bottles were gradually emptied, which is, in all likelihood, due to the design of the bottle (bottle walls are thicker and are therefore able to maintain the same level of rigidity).

Comparisons between suppliers uncovered an interesting diversity in drop volumes and repeatability of drop size. Differences in drop size were found to be related to nozzle parameters such as the outer diameter, platform width and inner diameter, although it is likely that there is a complex mathematical relationship between these parameters and drop volume. When suppliers were questioned about their choice of bottle/ nozzle manufacturer, many replied that rather than selecting on the basis of drop volume accuracy, factors such as bottle capacity, material and packing costs, level of automation in production, tamper-evident seals and service were considered more important. In some cases drop size may be assessed but this is generally done to ensure that an adequate number of drops will be available during the use of the product, rather than to determine the uniformity of dosage delivered. Contact was also made with European manufacturers of eye drop bottles and nozzles, such as Kerplas and Bunder Glas. Although quality control procedures and standards are high, it appears that bottles are produced to give drop sizes between $30 \mu \mathrm{l}$ and $50 \mu \mathrm{l}$, depending upon the requirements of their customers. 
Over the past few years MSD have started to address the potential problems associated with either large or variable drop volumes. A metered-dose unit, the Ocumeter, is now incorporated in all Timoptol bottles which should 'under laboratory conditions, produce a drop of $20 \mathrm{\mu l}^{\prime}{ }^{15}$ Unfortunately, this study has drawn attention to the fact that this is not the case - in all five bottles examined the drop volume was consistently greater than $20 \mu \mathrm{l}$, although the repeatability of drop size was amongst the best. Statistically significant variation in the volume of drops expelled from different bottles from the same supplier (MSD) was also evident. This may also be the case for other suppliers, although this was not experimentally confirmed. The experimental implications of such potential variation between bottles from the same suppliers would suggest that further study of parameters influencing drop volume using a larger number of bottles would be beneficial. The clinical implications of variation in drop size between bottles are detailed below.

It has been shown previously that volumes as small as $10 \mu l^{8}$ are quite adequate to produce a response, but differences in drop volume may cause variations in the level of drug response. In addition, the volume expelled from multi-dose bottles is far in excess of that required and may also present a greater risk of side effects through systemic absorption. At the present time, the Medicines Control Agency (MCA) in the UK and the Food and Drug Administration (FDA) in the USA have no official regulations for standardising drop volume, although the FDA does require that the drops dispensed in general use match the volumes used in clinical trials for that product. However, many researchers assessing the clinical efficacy of novel drugs still base results on the instillation of a number of drops rather than a specific volume.

In conclusion, the importance of the volume of drug instilled needs to be reiterated at all levels. Researchers should be aware of volume variations from eye drop containers and bottles and, where possible, start to use more accurate drug delivery equipment and techniques. Market suppliers of topical eye formulations should also be well informed of the minimum effective dose required for each drug. Thirdly, the manufacturers of bottles and nozzles need to be cognisant of the future potential requirements of their customers. Although it is appreciated that production of more accurate eye drop bottles producing smaller drops will have its initial difficulties, an attempt to improve the problem of large variability would be worthwhile before regulations are introduced to standardise drop volume.

\section{References}

1. Lund W. Pharmaceutical codex. 12th ed. London: Pharmaceutical Press, 1994:161-2.

2. File RR, Patton TF. Topically applied pilocarpine: human pupillary response as a function of drop size. Arch Ophthalmol 1980;98:112-5.

3. Gray RH. The influence of drop size on pupil dilatation. Eye 1991;5:615-9.

4. Patton TF. Pharmacokinetic evidence for improved ophthalmic drug delivery by reduction of instilled volume. J Pharm Sci 1977;66:1058-9.

5. Kessler C, Bleckmann H, Kleintges G. Influence of the strength, drop size and viscosity of metipranolol eye drops on the concentration of the substance in human aqueous humour. Graefes Arch Clin Exp Ophthalmol 1991;229:452-6.

6. Sugaya M, Nagataki S. Kinetics of topical pilocarpine in the human eye. Jpn J Ophthalmol 1978;22:127-41.

7. Brown RH, Hotchkiss ML, Davis EB. Creating smaller eyedrops by reducing eydropper tip dimensions. Am J Ophthalmol 1985;99:460-4.

8. Craig EW, Griffiths PG. Effect on mydriasis of modifying the volume of phenylephrine drops. Br J Ophthalmol 1991;75:222-3.

9. Fell JT. Surface and interfacial phenomena. In: Aulton ME, editor. Pharmaceutics: the science of dosage form design. Edinburgh: Churchill Livingstone, 1988:53-5.

10. German EJ, Hurst MA, Wood D. Eye drop container: a source of response variation? Ophthalmic Physiol Opt 1997;17:196-204.

11. Green $K$. The role of surfactants as bactericides in topical drug delivery. STP Pharm Sci 1992;2:34-8.

12. British Standard ISO-5725-1 Accuracy (trueness and precision) of measurement methods and results. Part 1. General principles and definitions, 1994.

13. Bland JM, Altman DG. Statistical methods for assessing agreement between two methods of clinical measurement. Lancet 1986;1:307-10.

14. Bland JM. An introduction to medical statistics. 1st ed. Oxford: Oxford University Press, 1993:118.

15. Personal communication, Technical Services, Merck Sharp Dohme Ltd. 